\title{
Immune-potentiation of Pneumococcal Capsular Polysaccharide Antigen using Albumin Microparticles
}

\author{
Bernadette D’Souza ${ }^{1}$, Prathap Nagaraja Shastri ${ }^{\star}$, Gabrielle Hammons ${ }^{3}$, Ellie Kim ${ }^{3}$, Lakshmi Prasanna Kolluru ${ }^{3}$, George M Carlone ${ }^{3}$, \\ Gowrisankar Rajam ${ }^{3^{*}}$ and Martin J D'Souza ${ }^{2}$
}

${ }^{1}$ McWhorter School of Pharmacy, Samford University, Birmingham, AL 35229, USA

${ }^{2}$ College of Pharmacy and Health Sciences, Nanotechnology Laboratory, Mercer University, Atlanta, GA 30341, USA

${ }^{3}$ Division of Bacterial Diseases, Centers for Disease Control and Prevention, Atlanta, GA 30333, USA

\begin{abstract}
Microparticles (MPs) offer several advantages as unique vaccine delivery system, including the ease to manufacture, targeted delivery of antigen payload, sustained antigen release and possible role as an immuneadjuvant. In this study, we evaluated albumin matrix for pneumococcal (Pnc) serotype specific capsular polysaccharide (PS) antigen MPs. Microencapsulation of Pnc PS was successful with a product yield of $>72 \%$. The MP size, 1-5 $\mu \mathrm{m}$, and negative zeta potential $(-26.5 \mathrm{mV})$ were optimized to ensure effective -uptake and presentation of Pnc PS antigens to immune cells. In mice, ST 19F and 23F MPs exhibited $>10$-fold increase $(P<0.01)$ in ST specific lgG response over PS in solution given with or without alum. Relatively higher immune response was observed for ST 6B MPs when compared to PS solution; however, ST6B PS solution along with alum resulted in an overall higher response when compared to ST6B MPs. Microencapsulation may offer a simple and effective mechanism for the immune-enhancement of poorly immunogenic antigens such as Pnc PS.
\end{abstract}

Keywords: Pneumonia; Vaccine; Microparticles; Spray drying; Pneumococcal polysaccharide

\section{Introduction}

The field of vaccine research has made great strides over the last decade. In the past, vaccines were usually comprised of either live attenuated or killed whole cells of target pathogens. Even though such vaccines most often provided an immune response comparable to natural infection in terms of duration and stimulation of immune system, they caused several unwanted local or systemic immunological complications that at times overweighed benefits. Furthermore, some of the chronic pathogens such as Mycobacterium tuberculosis, HIV and HCV require alternate vaccine strategies to address their intra-cellular parasitic nature [1-3]. Development of recombinant, synthetic or purified immunogenic component(s) instead of whole cells of pathogen has shown promising results and steered vaccine development away from live and attenuated vaccines. The poor immunogenic potential of some of these components necessitates the inclusion of an adjuvant to enhance or prolong an antigen specific immune response [3]. Addition of adjuvants to the vaccine formulations in turn resulted in adverse events and undesirable immunological complications [4].

Microparticle and nanoparticle based drug delivery system is an emerging technology which has shown great promise in the delivery of small molecule anti-cancer drugs and in particular, large molecule vaccines [5-8]. Microparticle vaccines have the ability to release antigens over time resulting in a robust immunity with a single dose thereby eliminating the need for multiple doses along with reducing the chances of vaccine dropouts [9]. As discussed earlier, duration and simulation of the immune response are two critical aspects of vaccine formulations [10,11]. Microparticle based vaccine delivery systems not only enhance the immune response by stimulating antigen presenting cells (APCs) that preferentially take up particulate antigens in comparison to antigen solution but also prolong antigen exposure by creating a depot effect resulting in modified/sustained release of antigens [12-14].

Polysaccharides (PS) on their own are known to be poor immunogens, since PS are T-independent antigens that do not result in long term memory response $[15,16]$. Conjugation of PS to a carrier protein converts them to T-dependent antigens that confer immunememory $[17,18]$. Streptococcus pneumoniae is a commensal bacterium found in the upper respiratory tract. Globally, pneumococcal disease due to this pathogen is prevalent in all ages [19-21]. The PS capsular chemistry and serological reactions has so far yielded greater than 103 pneumococcal (Pnc) serotypes (STs). There are conjugate and nonconjugate vaccines available for $S$. pneumoniae, including the 23 -valent non-conjugated polysaccharide vaccine, Pneumovax ${ }^{\mathrm{TN}}-23$ (Merck for adults > 65 years of age); and the 13-valent conjugate vaccine, Prevnar13 (PCV13, Wyeth, for patients of all ages). Pneumovax ${ }^{\mathrm{Tx}}-23$ is composed of purified Pnc capsular PS from 23 different STs. Pneumococcal capsular PS in their native form are poor immunogens $[22,23]$ therefore the 23-valent Pnc PS vaccine (Pneumovax) are less effective in children and most effective in adults $[23,24]$. The major limitation of Pnc PS vaccines is that it does not protect from Pnc STs that are not in the vaccine [25]. Due to large number of circulating Pnc STs, targeted removal of only a limited number of circulating STs by the current available vaccines creates a need for those STs that are not protected against in the marketed vaccines. Therefore, Pnc disease due to serotype deletion or replacement is inevitable [26]. Moreover, protein conjugation of PS antigens is a complicated process that increases the production cost of the vaccine. Considering the

*Corresponding author: Prathap Nagaraja Shastri, College of Pharmacy and Health Sciences, Nanotechnology Laboratory, Mercer University, Atlanta, GA 30341, USA, Tel: +1 678547 6353; Fax: +1 678547 64230; E-mail: prathap.shastri@ambrx.com

Gowrisankar Rajam, Division of Bacterial Diseases, Centers for Disease Control and Prevention, Atlanta, GA 30333, USA, Tel: 404639 2630; E-mail: rajamshanks@gmail.com

Received: May 22, 2018; Accepted: May 29, 2018; Published: June 05, 2018

Citation: D'Souza B, Shastri PN, Hammons G, Kim E, Kolluru LP, et al. (2018) Immune-potentiation of Pneumococcal Capsular Polysaccharide Antigen using Albumin Microparticles. J Pharmacovigil 6: 261. doi:10.4172/2329-6887.1000261

Copyright: (c) 2018 D'Souza B, et al. This is an open-access article distributed under the terms of the Creative Commons Attribution License, which permits unrestricted use, distribution, and reproduction in any medium, provided the original author and source are credited. 
potential benefits of particle-based drug delivery systems, we worked on development of novel microparticulate polysaccharide antigen prepared using a one-step spray drying technology. This manuscript summarizes microparticles effectiveness in resulting immunogenic response to $S$. pneumoniae serotype specific to capsular polysaccharide antigens.

\section{Materials and Methods}

\section{Preparation and characterization of microparticle formulation}

Microparticle formulation optimization: The lyophilized Pnc capsular PS (ST 6B, 19F and 23F; ATCC, Manassas, VA) were solubilized to make a stock solution of $2 \mathrm{mg} / \mathrm{mL}$ using de-ionized water with overnight agitation at $4^{\circ} \mathrm{C}$. Pnc PS MPs were formulated using a onestep spray drying technique [27]. Briefly, the MPs were prepared with mouse serum albumin (Thermo Fisher, Waltham, MA) matrix which was cross linked for 8 hours using glutaraldehyde. Sodium bisulphite was used to neutralize the excess glutaraldehyde, and $1 \% \mathrm{w} / \mathrm{v}$ chitosan was added to the resulting mixture to optimize the formulation. All the excipients added were Federal Drug Administration (FDA) Generally Recognized as Safe (GRAS) certified reagents. Pnc ST specific PS was maintained at $1 \%(\mathrm{v} / \mathrm{v})$ concentration and spray dried in a Buchi Mini Spray dryer (B-191, Buchi Corporation and New Castle, DE). In the spray dryer, the solution passes through an atomizer, which disperses the solution into a drop size spray in a controlled manner. The small sized drops encompass a large surface area and dries up quickly to form microparticles upon transient exposure to elevated temperatures.

The percentage product yield of MPs was calculated using the formula given below:

Percentage product yield $=$ Weight of microparticles obtained after spray drying $\times 100 /$ The total weight of all polymers along with antigen prior to spray drying.

Microparticle size distribution: The uptake of MPs by antigen presenting cells (APCs) including macrophages and dendritic cells can be greatly influenced by their particle size. Microparticle suspension $(10 \% \mathrm{w} / \mathrm{v})$ was made in $10 \mathrm{~mL}$ deionized water and sonicated for 1 minute. Particle size of the suspension was measured using Spectrex particle size counter (Spectrex Corporation, Redwood city, CA). The size range was determined for both blank and antigen loaded MPs.

Zeta potential: Achieving a well dispersed suspension without aggregation is key for successful administration of MP based formulation, and Zeta potential provides the measure of the same. Aqueous suspension $(10 \% \mathrm{w} / \mathrm{v})$ of Pnc PS loaded and blank MPs were tested for their Zeta potential using Malvern Zetasizer (Malvern Zetasizer Nano ZS, Malvern Instruments, Worcester, UK), adopting the methodology previously described [28].

Scanning electron microscopy of microparticle: The JEOL scanning electron microscopy was used to study the size, surface morphology, and uniformity of the MP formulation. Briefly, the formulations were evenly spread on metal stubs and coated with gold and dried. These gold-coated MPs were vacuum dried and then imaged under a nitrogen airflow stream.

\section{Antigen epitope integrity}

The epitope integrity of the antigen is critical for the success of formulation process and loading efficiency is crucial to calculate the dosage for in vivo studies. The phenol-sulphuric acid assay is generally used to quantify polysaccharides [29]. The use of chitosan (polysaccharide) as an excipient in this MP formulation warranted an alternate technique due to its interference in the PS quantification with the phenol-sulphuric acid technique. To address this challenge, an alternate technique that utilizes the principle of homologous competition between an antigen and specific antibody was implemented. Extraction of antigens from the MPs was done by treating with $50 \mathrm{mM}$ Tris $\mathrm{HCl}$, $10 \mathrm{mM}$ EDTA buffer, and followed by incubation with $0.2 \mathrm{~N} \mathrm{NaOH}$ and protease overnight at $37^{\circ} \mathrm{C}$. The diluted extract was further incubated with a Pnc reference standard serum, 89SF (1:20) overnight at $4^{\circ} \mathrm{C}$ with head to toe rotation. Additional tubes of standards were prepared containing the reference standard and range of concentrations of the Pnc ST PS (23F, 6B, and 19F) to generate Pnc Ps calibration graph. Following overnight incubations, the mixtures were centrifuged at 8000 $\times \mathrm{g}$ for 5 minutes and the supernatant was tested for Pnc ST specific IgG using microsphere based multiplex technique [30]. Pnc PS in MPs was calculated from the 89SF-Pnc PS calibration graph. Being an Pnc PS specific antibody capture technique, this also provides valuable information regarding the epitope integrity in the MP formulations.

\section{Test for microparticle cytotoxicity}

The epitope integrity of the antigen is critical for the success of formulation process and loading efficiency is crucial to calculate the dosage for in vivo studies. The phenol-sulphuric acid assay is generally used to quantify polysaccharides [29]. The use of chitosan (polysaccharide) as an excipient in this MP formulation warranted an alternate technique due to its interference in the PS quantification with the phenol-sulphuric acid technique. To address this challenge, an alternate technique that utilizes the principle of homologous competition between an antigen and specific antibody was implemented. Extraction of antigens from the MPs was done by treating with $50 \mathrm{mM}$ Tris $\mathrm{HCl}$, $10 \mathrm{mM}$ EDTA buffer, and followed by incubation with $0.2 \mathrm{~N} \mathrm{NaOH}$ and protease overnight at $37^{\circ} \mathrm{C}$. The diluted extract was further incubated with a Pnc reference standard serum, 89SF (1:20) overnight at $4^{\circ} \mathrm{C}$ with head to toe rotation. Additional tubes of standards were prepared containing the reference standard and range of concentrations of the Pnc ST PS (23F, 6B, and 19F) to generate Pnc Ps calibration graph. Following overnight incubations, the mixtures were centrifuged at 8000 $\times \mathrm{g}$ for 5 minutes and the supernatant was tested for Pnc ST specific IgG using microsphere based multiplex technique [30]. Pnc PS in MPs was calculated from the 89SF-Pnc PS calibration graph. Being an Pnc PS specific antibody capture technique, this also provides valuable information regarding the epitope integrity in the MP formulations.

\section{Test for microparticle cytotoxicity}

Blank MPs were tested for cytotoxicity before animal immunization. Cell cytotoxicity studies were done with blank MPs using murine macrophages, RAW 246.7. About $2 \times 10^{4}$ cells $/ \mathrm{mL}$ of RAW 246.7 macrophage cells were plated in a 96 well plate. Microparticle suspension in the concentration range of $10-1000 \mu \mathrm{g} / \mathrm{mL}$ was added on to the cells. Atropine sulphate was used as the positive control at a concentration of $10 \mathrm{mg} / \mathrm{mL}$. The plates were incubated for 12 hours at $37^{\circ} \mathrm{C}$ with $5 \% \mathrm{CO}_{2}$. Upon completion of incubation, about $20 \mu \mathrm{L} /$ well of Alamar blue dye (Life Technologies, Grand Island, NY) was added and the plate was read at $490 \mathrm{~nm}$ in a microplate reader. Alamar blue is a redox indicator and color change from blue to purple to red is directly proportional to the cellular respiratory activity and thus, viability.

\section{In vivo immunization studies}

Mouse strains: Female Swiss Webster mice (Charles River Laboratories, Wilmington, MA) 6 to 10 weeks of age were used in 
Citation: D'Souza B, Shastri PN, Hammons G, Kim E, Kolluru LP, et al. (2018) Immune-potentiation of Pneumococcal Capsular Polysaccharide Antigen using Albumin Microparticles. J Pharmacovigil 6: 261. doi:10.4172/2329-6887.1000261

Page 3 of 6

this study. All experiments were approved by the Institutional Animal Care and Use Committee (IACUC) and conducted according to the institutional ethical guidelines for animal experiments and safety guidelines. Alum $\left(\mathrm{Al}_{2} \mathrm{OH}\right)$ was used as an adjuvant. Each Pnc serotype testing, six groups ( $n=10$ /group) of mice were included. This includes, control groups that received blank MPs, and Pnc ST specific PS in solution with and without alum. Apart from Pnc PS MPs, another group of animals received Pnc PS in solution with blank MPs separately to test the role of MPs as an external adjuvant. Two doses of $50 \mu \mathrm{g}$ of MPs/pure Pnc PS were administered via subcutaneous administration with a 15 day dosing interval. Blood was collected on day 0 prior to immunizations, at day 14 and one week after the last dose.

\section{Pnc ST specific IgG quantification}

Serum samples were analyzed using multiplex Luminex technique [30] to specifically detect IgG with Pnc STs with some modification. The filter plates (MABVN1250, Millipore Corp, Billerica, MA) were pre-wet with $100 \mu \mathrm{L}$ assay buffer $(0.1 \%$ BSA in PBS). Serum dilutions were carried out in a 96-well round bottom titer plate (Dilution plate, CLS3799, Sigma, St. Louis, MO). Test serum samples were diluted 2 -fold for 7 dilutions starting at 1/50. For reagent controls, each assay included buffer blanks. Replicates were maintained for unknown serum and blanks. The Pnc ST specific PS conjugated fluorescent microspheres were added to the plates at $25 \mu \mathrm{L} / 5000$ beads/well/PS after $2 \mathrm{X}$ PBS prewash. To this, $25 \mu \mathrm{L} /$ well of serum sample was transferred from the dilution plate and incubated at $37^{\circ} \mathrm{C}$ for 30 minutes with agitation. After 3 washes with $100 \mu \mathrm{L} /$ well PBS, a secondary PE labeled antimouse antibody (Moss Pasadena, MD), was added and incubated for 20 minutes with agitation. On completion, plates were washed three times with $100 \mu \mathrm{L} /$ well PBS and the beads were resuspended in $130 \mu \mathrm{L}$ of luminex sheath fluid before reading in the luminex reader (L200, Luminex Corp, Austin, TX).

\section{Statistical Analysis}

Animal studies were carried out in two independent cycles. Masterplex QT (Miraibio, Hitachi, Irvine, CA) was used for the analysis of mice serum IgG data extracted from the luminex reader. One-way ANOVA and Tukey test was performed on in vivo data using graph pad Prism. For all the data collected; results were tabulated as significant at $p$ values $<0.05$. In the absence of reference standard calibrated for mice sera, animal experiment groups and study time points were compared based on their respective Median Fluorescence Intensity (MFI) and immune response was expressed as fold changes.

\section{Results}

\section{Characterization of albumin microparticles}

The percentage product yield was measured as $76 \% \pm 3.54$ and $75 \%$ \pm 4.18 for blank and antigen loaded MPs, respectively. The size of the MPs ranged from 1-2 $\mu \mathrm{m}$ in majority with overall size range of 1-5 $\mu \mathrm{m}$ (Table 1). No significant difference was seen in the particle size between the blank and the Ps antigen loaded MPs. The zeta potential was found to be $-24 \mathrm{mV}$ and $-26.5 \mathrm{mV}$ for blank and antigen loaded MPs respectively (Table 1). Scanning electron microscopy revealed uniform spherical shape in MPs with each particle separated distinctly (Figure 1).

\section{Loading efficiency of microparticle and antigen epitope integrity}

Antigen epitope integrity was verified by quantifying Pnc ST

\begin{tabular}{|c|c|c|c|c|}
\hline \multirow{2}{*}{ Parameter } & \multirow{2}{*}{ Blank particle } & \multicolumn{3}{|c|}{ Antigen loaded particle } \\
\cline { 3 - 5 } & & ST 6B & ST 19F & ST23F \\
\hline Product yield (\%) & $76 \pm 3.54$ & \multicolumn{3}{|c|}{$75 \pm 4.18$} \\
\hline Particle size range & $1-5 \mu \mathrm{m}$ & \multicolumn{3}{|c|}{$1-5 \mu \mathrm{m}$} \\
\hline Zeta potential & $-24 \mathrm{mv}$ & \multicolumn{3}{|c|}{$-26.5 \mathrm{mv}$} \\
\hline Size distribution & $1 \%$ & & $1 \%$ & $1 \%$ \\
$<1.0 \mu \mathrm{m}$ & $70 \%$ & $1 \%$ & $70 \%$ & $70 \%$ \\
$1.0-2.0 \mu \mathrm{m}$ & $20 \%$ & $75 \%$ & $20 \%$ & $20 \%$ \\
$2.1-3.0 \mu \mathrm{m}$ & $10 \%$ & $15 \%$ & $10 \%$ & $10 \%$ \\
$3.0-4.0 \mu \mathrm{m}$ & $8 \%$ & $10 \%$ & $8 \%$ & $8 \%$ \\
$4.1-5.0 \mu \mathrm{m}$ & $1 \%$ & $8 \%$ & $1 \%$ & $1 \%$ \\
$>5.0 \mu \mathrm{m}$ & & $1 \%$ & $80.76 \%$ & $83.64 \%$ \\
\hline Antigen loading & NA & $72.5 \%$ & & \\
efficiency ${ }^{\mathrm{a}}$ & & \multicolumn{3}{|c|}{} \\
\hline
\end{tabular}

$\mathrm{a}=$ Antigen loading efficiency also indicates epitope integrity as this was calculated based on the immune reactivity of the ST PS eluted from the MPs with the SP specific PS antibodies

Table 1: Microparticles yield and characteristics.

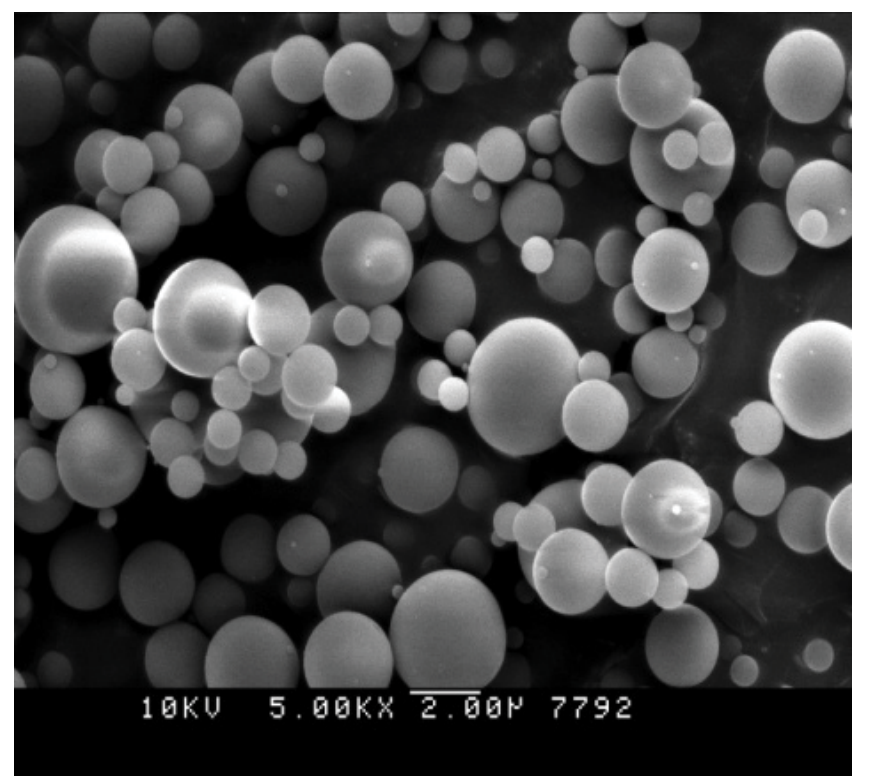

Figure 1: Scanning Electron Microscopy (SEM). SEM image of the polysaccharide antigen loaded microparticles. The smooth surface morphology and spherical size of particles were a result of the formulation optimization. The scale at the bottom shows the $2 \mu \mathrm{m}$ size which is representative of majority of microparticles.

specific PS using Luminex as described in section 2.2. 89SF, the reference serum has ST specific IgG assignments that react to Pnc ST specific Ps. The quantification assay demonstrated $72.5 \%, 83 \%$, and $80 \%$ bioactivity retention for $6 \mathrm{~B}, 23 \mathrm{~F}$, and $19 \mathrm{~F}$, respectively (Table 1 ). Therefore, this methodology confirms that the formulation process has no major impact on the epitope integrity of the Pnc PS antigen.

\section{In vitro microparticle cell cytotoxicity}

In vitro cell toxicity test was performed to determine the effect of MPs on eukaryotic cells using murine macrophages, RAW 246.7 model. Data presented in Figure 2 show that MPs have little or no toxic effect $(\mathrm{p}<0.05)$ on RAW 246.7 cells.

\section{In vivo immunization studies}

Bioactive levels of Pnc STs 19F and 23F were determined from serum of mice dosed with the MPs containing antigens. Both ST specific PS MP treatment groups resulted in an apparent greater immune response with $19 \mathrm{~F}$ and $23 \mathrm{~F}$ MPs resulting in 10- and 20-fold 
statistically significant increase in immune response as compared to other groups including antigen solution control and with adjuvant alum (Figure $3 \mathrm{a}$ and $3 \mathrm{~b}$ ). Although, in case of $6 \mathrm{~B}$ serotype, vaccine solution with alum showed significantly better immune response when compared to 6B MPs. However, when compared to $6 \mathrm{~B}$ solution alone, MP vaccine produced higher immune response (Figure $3 \mathrm{c}$ ).

\section{Discussion}

This research project was focused on optimizing a novel particulate formulation to improve the efficacy of pneumococcal antigens in a

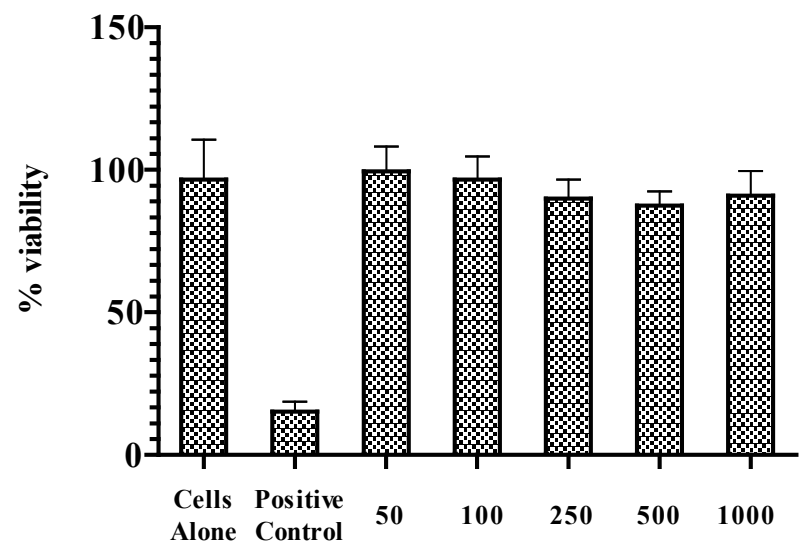

Microparticle Concentration $(\mu \mathrm{g} / \mathrm{mL})$

Figure 2: Cell Cytotoxicity Studies. The Alamar blue assay was used to determine the toxicity of the microparticles containing polysaccharide antigens. The microparticles were found to be non-cytotoxic in the concentration range of $50-1000 \mu \mathrm{g} / \mathrm{ml}$. After 24 hours of incubation more than $80 \%$ of the cells were viable at all the tested concentrations. The positive control showed cytotoxicity with viability of less than $20 \%$. Error bars=Standard deviation.

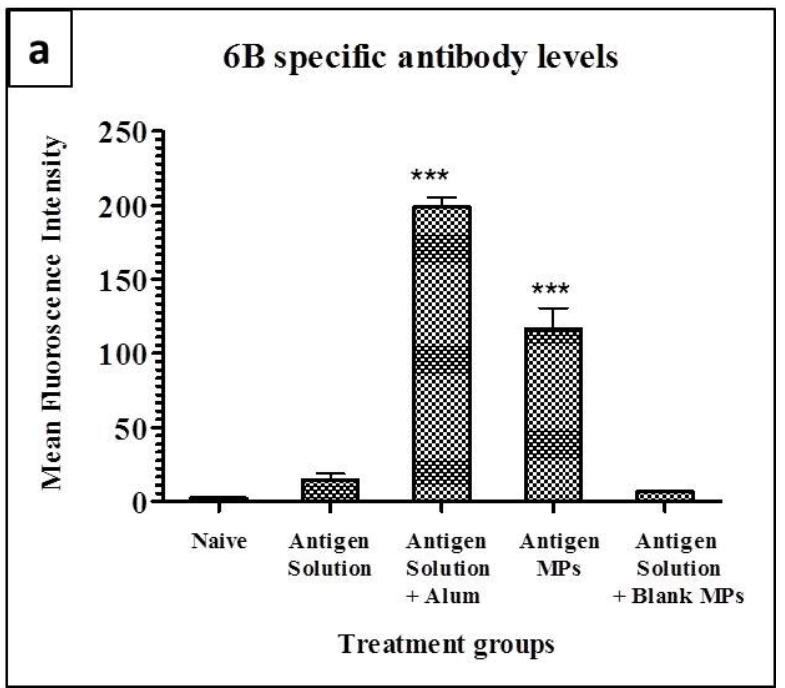

Figure 3a: Immunogenicity of micro articulated pneumococcal polysaccharide antigen compared to alum. Serotypes $6 \mathrm{~B}, 19 \mathrm{~F}$ and $23 \mathrm{~F}$ specific antibody levels in serum samples from mice receiving i) buffer alone, ii) polysaccharide antigen in solution (PS soln), iii) polysaccharide antigen with alum (PS Soln + alum), microparticles of polysaccharide antigen (PS MPs), and Polysaccharide antigen with blank MSA particles (PS soln+blk MPs). Serotype 6B specific serum antibody levels showed that while $6 \mathrm{~B}$ loaded microparticles resulted in better immune response to the solution form of polysaccahride antigen, the PS solution administered with alum resulted in a higher immune response. ${ }^{* *}<$ 0.01 and $\left.{ }^{* * *} p<0.001\right)$.

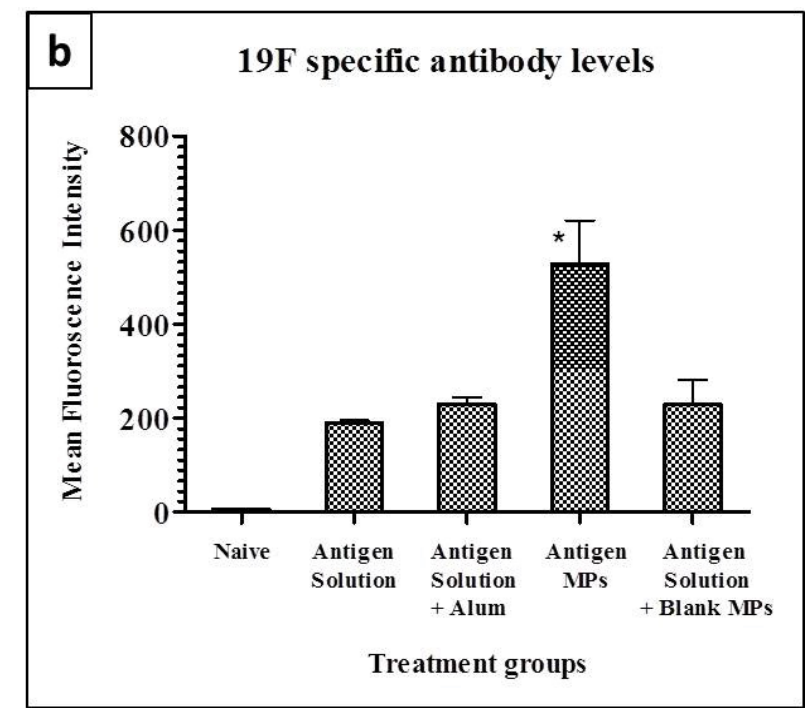

Figure 3b: Serotype 19F specific serum antibody levels showed that microparticles of the ST $19 \mathrm{~F}$ polysaccharide antigens resulted in a significantly higher immune reponse $\left({ }^{*} p<0.05\right)$ when compared to all other groups.

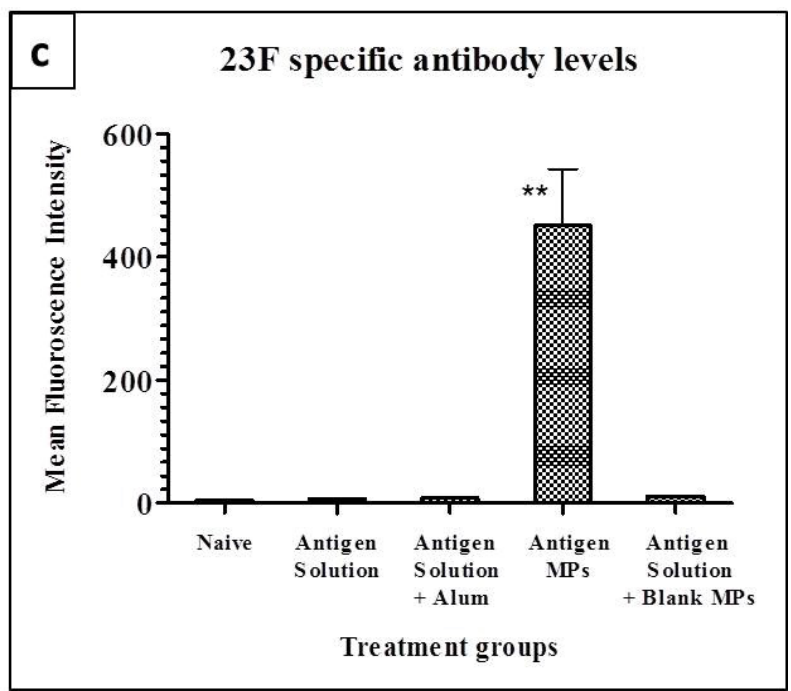

Figure 3c: Serotype 23F specific serum antibody levels showed that microparticles of the ST $23 \mathrm{~F}$ polysaccharide antigens resulted in the significantly higher immune reponse $\left({ }^{* *} p<0.01\right)$ when compared to all other groups.

vaccine as well as to extend vaccine coverage $[18,22]$. We formulated pneumococcal PS MPs having a homogenous size range of 1-5 $\mu \mathrm{m}(90 \%$ MPs 1-2 $\mu \mathrm{m}$ ). The MPs at this size range ensures effective uptake and processing of Pnc PS by antigen presenting cells (APC) and dendritic cells (DC) of the immune system. Buchi-190 was used to spray dry the MPs containing the ST specific Pnc Ps. Spray drying is a commonly used technique to prepare small to medium sized batches of particulate delivery systems including antigens and large molecules [31,32]. This technique ensures the formulation of MPs with size and surface properties critical for the uptake and stimulation of innate immune cells. Another critical physical characteristic of MPs is its ability to stay non-aggregated. Aggregated MPs lead to gross increase in their size leading to a sharp decline in the immunological benefits. The surface charge of MPs made by the spray drying technique were $> \pm 30 \mathrm{mV}$ 
which would impede aggregation and make the MPs more suitable for uptake by immune cells for an effective vaccination. In this study, optimal formulation condition yielded Pnc PS MPs at $-25 \mathrm{mV}$ in water that would discourage particle aggregation resulting in heightened immunological benefits. The smooth spherical surface morphology of the MPs as seen in SEM images suggests the possibility of uniform release of antigens in vivo. This would allow slow and sustained release of antigen encapsulated within the MP when taken up by antigen presenting cells (APCs) and dendritic cells (DCs).

A luminex-based bioassay was developed to quantify Pnc PS in MPs as the use of polysaccharide excipients impeded the use of the traditional phenol-sulfuric acid assay for PS quantification. This bioassay was able to quantify the Pnc ST specific PS antigens as well as determine the bioactivity and epitope integrity of PS in MPs after formulation. In its multiplex format, this bioassay also offers the potential for the simultaneous quantification of multivalent MP formulation. Using this technique, the MP loading efficiency for all 3 Pnc ST was found to be $>70 \%$. This also reiterates the epitope integrity of the encapsulated Pnc PS as the analytical basis of this bioassay is the reaction between the antibody and the complementary epitope on the Pnc PS. This proves that the MP formulation technique used in this study is effective and has minimal impact on the epitope integrity of the antigen in MPs. Encouraged by the successful encapsulation of Pnc ST Ps resulting in MPs with desirable physicochemical properties and safety profiles, the Pnc PS MPs were compared for their immunogenicity in vivo to PS in solution. The animal experiments yielded a superior immune response with $\geq 10$-fold increase in Pnc ST specific IgG for $19 \mathrm{~F}$ and $23 \mathrm{~F}$ in MPs as compared to the PS in solution. The serotype $6 \mathrm{~B}$ in the MPs did show a robust IgG response, however the increase was not as significant as the $19 \mathrm{~F}$ and $23 \mathrm{~F}$ serotypes in MPs versus their solutions. This was not surprising since serotype $6 \mathrm{~B}$ is one of the least immunogenic in the spectrum of serotypes used in the 23-valent Pnc PS vaccine (Pneumovax) formulation for adults. Further, ST 6B in the Prevnar-13 pneumococcal conjugate-vaccine has also been reported to be less immunogenic as compared to the other STs in the vaccine [33-35]. Higher loading concentration and/ or alternate MP formulation strategies may help to improve the PS 6B immunogenicity.

The enhanced ST specific immunity can be attributed to the optimized size and surface morphology of the PS MPs that is critical for uptake by immune responsive cells. It has been shown that 2- 8 $\mu \mathrm{m}$ MPs loaded with hepatitis B surface antigen elicited higher IgG response given intra muscularly [28]. Kalakinidis et al., suggested that MPs with a size distribution around $500 \mathrm{~nm}$ and up to roughly 3- $5 \mu \mathrm{m}$ were taken up mainly by phagocytosis and promote humoral immune response [36]. As suggested by Balasse et al., the 1- $5 \mu \mathrm{m}$ size for the optimized polysaccharide MPs help in increasing duration of exposure to DCs resulting in an increase of antigen specific immune response due to particulate nature over solution form [37].

Interestingly, blank MPs failed to exert an adjuvant effect on the Pnc PS solution form. This reiterates that steady state release of MPs entrapped antigens is crucial for the continuous insult of the immune system resulting in improved immune response. Several researchers have reported the usefulness of the blank MPs as a non-specific adjuvant but there are multiple factors that may govern this response. This property could be determined by the chemistry of the polymer or the reactivity of the polymer with the animal species tested to name a few. In one study, ionic polyphosphazene or PLGA were shown to have demonstrable immune adjuvant activity on their own [38,39].
We have shown that optimized microparticles with serotype specific antigens provide an opportunity for formulating pneumococcal vaccines that can be customized to specific geographical and demographic requirements using a cost-effective manufacturing process. This process is vital for diseases due to multiple virulent serotypes/serogroups in circulation. In our earlier studies, oral MPs with several vaccines including pertussis, typhoid and tuberculosis vaccine have shown promising outcome in animal models [40-43]. The present study aligns with the previous observations and holds promise as the next generation vaccine formulation strategy for pneumococcal disease. Pnc PS MPs have been extremely efficacious in humoral immune induction and ST specific antibody response in vivo. Additional studies to deduce the effect of MP immunization on the cellular immune response, Pnc carriage and disease in vivo is recommended to consolidate these MPs as Pnc PS vaccine delivery systems.

\section{References}

1. Cascalho M, Balin SJ, Platt JL (2017) The mutable vaccine for mutable viruses Immunotherapy 9: 659-667.

2. O'Hagan DT, Singh M (2003) Microparticles as vaccine adjuvants and delivery systems. Expert Rev Vaccines 2: 269-283.

3. Gause KT, Wheatley AK, Cui J, Yan Y, Kent SJ, et al. (2017) Immunological principles guiding the rational design of particles for vaccine delivery. ACS Nano 11: 54-68.

4. Petrovsky N (2015) Comparative safety of vaccine adjuvants: A summary of current evidence and future needs. Drug Saf 38: 1059-1074.

5. Kunda NK, Alfagih IM, Dennison SR, Tawfeek HM, Somavarapu S, et al. (2015) Bovine serum albumin adsorbed PGA-co-PDL nanocarriers for vaccine delivery via dry powder inhalation. Pharm Res 32: 1341-1353.

6. Gala RP, D'Souza M, Zughaier SM (2016) Evaluation of various adjuvan nanoparticulate formulations for meningococcal capsular polysaccharidebased vaccine. Vaccine 34: 3260-3267.

7. Kolluru LP, Rizvi SA, D'Souza M, D'Souza MJ (2013) Formulation development of albumin based theragnostic nanoparticles as a potential delivery system for tumor targeting. J Drug Target 21: 77-86.

8. Tawde SA, Chablani L, Akalkotkar A, D'Souza MJ (2016) Evaluation of microparticulate ovarian cancer vaccine via transdermal route of delivery. J Control Release 235: 147-154.

9. Guarecuco R, Lu J, McHugh KJ, Norman JJ, Thapa LS, et al. (2017) Immunogenicity of pulsatile-release PLGA microspheres for single-injection vaccination. Vaccine.

10. Johansen P, Storni T, Rettig L, Qiu Z, Der-Sarkissian A, et al. (2008) Antigen kinetics determines immune reactivity. PNAS 105: 5189-5194.

11. Silva AL, Rosalia RA, Varypataki E, Sibuea S, Ossendorp F, et al. (2015) Poly(lactic-co-glycolic-acid)-based particulate vaccines: particle uptake by dendritic cells is a key parameter for immune activation. Vaccine 33: 847-854.

12. Brito LA, Chan M, Shaw CA, Hekele A, Carsillo T, et al. (2014) A cationic nanoemulsion for the delivery of next-generation RNA vaccines. Mol Ther 22 2118-2129.

13. Storni T, Kundig TM, Senti G, Johansen P (2005) Immunity in response to particulate antigen-delivery systems. Adv Drug Deliv Rev 57: 333-355.

14. Ubale RV, Gala RP, Zughaier SM, D'Souza MJ (2014) Induction of death receptor CD 95 and co-stimulatory molecules CD80 and CD86 by meningococcal capsular polysaccharide-loaded vaccine nanoparticles. AAPS J 16: 986-993.

15. Moffitt K, Malley R (2016) Rationale and prospects for novel pneumococcal vaccines. Hum Vaccin Immunother 12: 383-392.

16. Mond JJ (1995) T cell independent antigens. Curr Opin Immunol 7: 349-354

17. Broker M, Berti F, Schneider J, Vojtek I (2017) Polysaccharide conjugate vaccine protein carriers as a "neglected valency" - Potential and limitations. Vaccine 35: 3286-3294.

18. Seo JY, Seong SY, Ahn BY, Kwon IC, Chung H, et al. (2002) Cross-protective 
Citation: D'Souza B, Shastri PN, Hammons G, Kim E, Kolluru LP, et al. (2018) Immune-potentiation of Pneumococcal Capsular Polysaccharide Antigen using Albumin Microparticles. J Pharmacovigil 6: 261. doi:10.4172/2329-6887.1000261

immunity of mice induced by oral immunization with pneumococcal surface adhesin a encapsulated in microspheres. Infect Immun 70: 1143-1149.

19. Cilloniz C, Civljak R, Nicolini A, Torres A (2016) Polymicrobial communityacquired pneumonia: An emerging entity. Respirology 21: 65-75.

20. Cilloniz C, Martin-Loeches I, Garcia-Vidal C, San Jose A, Torres A (2016) Microbial Etiology of Pneumonia: Epidemiology, Diagnosis and Resistance Patterns. Int J Mol Sci 17: 2120.

21. Fedson DS, Scott JA (1999) The burden of pneumococcal disease among adults in developed and developing countries: what is and is not known. Vaccine 17 Suppl 1: S11-S18

22. Flanagan MP, Michael JG (1999) Oral immunization with a Streptococcal pneumoniae polysaccharide conjugate vaccine in enterocoated microparticles induces serum antibodies against type specific polysaccharides. Vaccine 17 72-81.

23. O'Brien KL, Wolfson LJ, Watt JP, Henkle E, Deloria-Knoll M, et al. (2009) Burden of disease caused by Streptococcus pneumoniae in children younger than 5 years: global estimates. Lancet 374: 893-902.

24. De Roux A, Schmole-Thoma B, Siber GR, Hackell JG, Kuhnke A, et al. (2008) Comparison of pneumococcal conjugate polysaccharide and free polysaccharide vaccines in elderly adults: conjugate vaccine elicits improved antibacterial immune responses and immunological memory. Clin Infect Dis 46: 1015-1023.

25. Prevnar 13 Product Insert. Pfizer.

26. Weinberger DM, Malley R, Lipsitch M (2011) Serotype replacement in disease after pneumococcal vaccination. Lancet 378: 1962-1973.

27. Gayakwad SG, Bejugam NK, Akhavein N, Uddin NA, Oettinger CE, et al (2009) Formulation and in vitro characterization of spray-dried antisense oligonucleotide to NF-kB encapsulated albumin microspheres. J Microencapsul 26: $692-700$

28. Bhowmik T, D'Souza B, Shashidharamurthy $R$, Oettinger C, Selvaraj $P$ et al. (2011) A novel microparticulate vaccine for melanoma cancer using transdermal delivery. Journal of Microencapsul 28: 294-300.

29. Masuko T, Minami A, Iwasaki N, Majima T, Nishimura S, et al. (2005) Carbohydrate analysis by a phenol-sulfuric acid method in microplate format. Anal Biochem 339: 69-72.

30. Lal G, Balmer P, Stanford E, Martin S, Warrington R, et al. (2005) Development and validation of a nonaplex assay for the simultaneous quantitation of antibodies to nine Streptococcus pneumoniae serotypes. Journal of immunological methods 296: 135-147.

31. Anish C, Khan N, Upadhyay AK, Sehgal D, Panda AK (2014) Delivery of polysaccharides using polymer particles: implications on size-dependent immunogenicity, opsonophagocytosis, and protective immunity. Mol Pharm 11 : 922-937.

32. D'Souza B, Bhowmik T, Shashidharamurthy R, Oettinger C, Selvaraj $P$, et al. (2012) Oral microparticulate vaccine for melanoma using M-cell targeting. Journal of Drug Targeting 20: 166-173.

33. Jacobson RM, Poland GA (2002) The pneumococcal conjugate vaccine. Minerva pediatrica 54: 295-303.

34. Knuf M, Szenborn L, Moro M, Petit C, Bermal N, et al. (2009) Immunogenicity of routinely used childhood vaccines when coadministered with the 10-valent pneumococcal non-typeable Haemophilus influenzae protein D conjugate vaccine (PHiD-CV). Pediatr Infect Dis J 28 (4 Suppl): S97-S108.

35. Cadeddu C, De Waure C, Gualano MR, Di Nardo F, Ricciardi W (2012) 23-valent pneumococcal polysaccharide vaccine (PPV23) for the prevention of invasive pneumococcal diseases (IPDs) in the elderly: is it really effective? JPMH 53: 101-103.

36. Kalkanidis M, Pietersz GA, Xiang SD, Mottram PL, Crimeen-Irwin B, et al (2006) Methods for nano-particle based vaccine formulation and evaluation of their immunogenicity. Methods 40: 20-29.

37. Balasse E, Odot J, Gatouillat G, Andry MC, Madoulet C (2008) Enhanced immune response induced by BSA loaded in hydroxyethylstarch microparticles. Int J Pharm 353: 131-138.

38. Andrianov AK, Marin A, Roberts BE (2005) Polyphosphazene Polyelectrolytes: A Link between the Formation of Noncovalent Complexes with Antigenic Proteins and Immunostimulating Activity. Biomacromolecules 6: 1375-1379.

39. Oyewumi MO, Kumar A, Cui Z (2010) Nano-microparticles as immune adjuvants: correlating particle sizes and the resultant immune responses. Expert Rev Vaccines 9: 1095-1079.

40. Dounighi NM, Shahcheraghi F, Razzaghi-Abyaneh M, Nofeli M, Zolfagharian H (2017) A New Vaccine Delivery Vehicle and Adjuvant Candidate: Bordetella Pertussis Inactivated Whole Cells Entrapped in Alginate Microspheres. Curr Pharm Des 23: 2665-2672.

41. Mutwiri G, Bowersock TL, Babiuk LA (2005) Microparticles for oral delivery of vaccines. Expert Opin Drug Deliv 2: 791-806.

42. Uddin AN, Bejugam NK, Gayakwad SG, Akther P, D'Souza MJ (2009) Ora delivery of gastro-resistant microencapsulated typhoid vaccine. J Drug Target 17: 553-560.

43. Yeboah KG, D'Souza M J (2009) Evaluation of albumin microspheres as ora delivery system for Mycobacterium tuberculosis vaccines. J Microencapsul 26: 166-79. 